\title{
Producción de pepino (Cucumis sativus L.) bajo invernadero: correlaciones entre variables
}

\section{Cucumber (Cucumis sativus L.) production under greenhouse conditions: co- rrelations among variables}

\author{
Karla Chacón-Padilla ${ }^{1}$ \\ Instituto Tecnológico de Costa Rica, Costa Rica \\ José Eladio Monge-Pérez ${ }^{2}$ \\ Universidad de Costa Rica, Costa Rica
}

\begin{abstract}
Resumen. Se establecieron correlaciones de Pearson para 14 genotipos de pepino producidos bajo invernadero, entre siete variables cuantitativas: longitud del fruto $(\mathrm{cm})$, diámetro del fruto $(\mathrm{mm})$, peso del fruto $(\mathrm{g})$, número de frutos por planta, rendimiento total y comercial $(\mathrm{kg} / \mathrm{m} 2)$ y porcentaje de sólidos solubles totales ( ${ }^{\circ}$ Brix). Se presentaron ocho correlaciones de Pearson evaluadas en los 14 genotipos que fueron altas $(r \geq 0,66)$ y con significancia estadística ( $\mathrm{p} \leq 0,05)$, en cuyo caso se obtuvieron las regresiones lineales: longitud y peso del fruto $(\mathrm{r}=0,92)$, diámetro y peso del fruto $(\mathrm{r}=0,66)$, peso del fruto y número total de frutos por planta $(\mathrm{r}=-0,84)$, diámetro del fruto y número total de frutos por planta $(\mathrm{r}=-$ $0,77)$, diámetro del fruto y porcentaje de sólidos solubles totales $(\mathrm{r}=0,69)$, número total de frutos por planta y porcentaje de sólidos solubles totales $(\mathrm{r}=-0,67)$, longitud del fruto y número total de frutos por planta $(\mathrm{r}=-0,72)$ y rendimiento total y comercial $(\mathrm{r}=0,81)$. Además, se obtuvieron otras cuatro correlaciones altas y con significancia estadística, según el tipo de pepino (largo, mediano o pequeño): rendimiento total y comercial, número total de frutos por planta y rendimiento total, número total de frutos por planta y rendimiento comercial y, únicamente para el pepino tipo pequeño, entre diámetro del fruto y porcentaje de sólidos solubles totales. Se concluye que el tipo de pepino incluido en el análisis influye de manera importante en el resultado de las correlaciones. El número total de frutos por planta se debe considerar una característica esencial en el fitomejoramiento para el rendimiento en pepino.
\end{abstract}

Palabras clave. Cucumis sativus, correlación de Pearson, regresión lineal, rendimiento, número de frutos por planta.

\begin{abstract}
For 14 cucumber genotypes grown under greenhouse conditions the researchers estimated Pearson correlations between seven quantitative variables: fruit length $(\mathrm{cm})$, fruit width $(\mathrm{mm})$, fruit weight $(\mathrm{g})$, number of fruits per plant, total and commercial yield $(\mathrm{kg} / \mathrm{m} 2)$, and percentage of total soluble solids ( ${ }^{\circ}$ Brix). Among the 14 evaluated genotypes there were eight Pearson correlations that were high $(r \geq 0,66)$ and statistically significant $(p \leq 0,05)$. In those cases the linear regression statistics were calculated: fruit length and weight $(\mathrm{r}=0,92)$; fruit width and weight $(\mathrm{r}=0,66)$; fruit weight and number of fruits per plant $(\mathrm{r}=-0,84)$; fruit width and number of fruits per plant $(\mathrm{r}=-0,77)$; fruit width and percentage of total soluble solids $(\mathrm{r}=0,69)$; number of fruits per plant and percentage of total soluble solids $(\mathrm{r}=-0,67)$; fruit length and number of fruits per plant $(\mathrm{r}=-0,72)$; and total and commercial yield $(\mathrm{r}=0,81)$. Statistically significant correlations were also observed in four additional cases, not across all genotypes but estimated according to the type of cucumber (long, medium or small): total and commercial yield; number of fruits per plant and total yield; number of fruits per plant and commercial yield. And, only for the small cucumber type, there were significant correlations between fruit width and percentage of total soluble solids. It is concluded that the cucumber type included in the analysis has a large effect in the correlation results. The number of fruits per plant should be considered an essential characteristic for yield breeding in cucumber.
\end{abstract}

Keywords. Cucumis sativus, Pearson correlation, linear regression, yield, number of fruits per plant.

\footnotetext{
${ }^{1}$ Ingeniera agrónoma, graduada del Instituto Tecnológico de Costa Rica. Dirección electrónica: kchpadilla@hotmail.com, Desamparados, Costa Rica. 2Ingeniero agrónomo, Investigador de la Estación Experimental Agrícola Fabio Baudrit Moreno, docente de la Sede de Guanacaste, Universidad de Costa Rica. Dirección electrónica: melonescr@yahoo.com.mx
} 


\section{Introducción}

El pepino Cucumis sativus L. es una especie importante de la familia Cucurbitaceae y corresponde a uno de los cultivos hortícolas más antiguos, pues fue domesticado en India hace unos 3000 años (Deepa, Hadimani, Hanchinamani, Shet, Koulgi, \& Ashok, 2018). Sus frutos inmaduros se consumen crudos en forma de ensalada, cocinados como hortaliza, o en conserva, y constituyen una fuente rica en minerales como calcio, fósforo, magnesio, potasio, manganeso y zinc; en vitaminas $\mathrm{B}$ y C; y en fitonutrientes como betacaroteno, betaxanteína y luteína (Deepa, y otros, 2018; Nwofia, Amajuoyi, \& Mbah, 2015).

El pepino es una especie termofílica y susceptible al frío, pues requiere una temperatura superior a $20{ }^{\circ} \mathrm{C}$. Se desarrolla mejor entre 25 y $29^{\circ} \mathrm{C}$ y con alta intensidad lumínica (Soleimani, Ahmadikhah, \& Soleimani, 2009; Pal, Sharma, Das, \& Pandav, 2017; Sharma, Kumar, Chatterjee, \& Sharma, 2018). En condiciones de invernadero, la planta de pepino crece rápidamente, por lo que necesita un adecuado abastecimiento de agua y nutrientes, dado que la tasa de absorción de nutrientes en esas condiciones es muy alta (Soleimani, y otros, 2009).

Las variedades ginoicas de pepino (100 $\%$ de flores femeninas) usualmente son más productivas. Generan frutos con una cáscara más lisa en comparación con las variedades monoicas (flores masculinas y femeninas) (Soleimani, y otros, 2009). Los pepinos partenocárpicos no producen semillas, porque sus frutos no requieren polinización. Además, tienden a producir frutos en forma más precoz, con un cuaje más concentrado, y con un rendimiento más alto (Soleimani y otros, 2009).

Entre los tipos de pepino, que se producen en invernadero, se destaca el tipo "slicer", cuyos frutos cuentan con un tamaño mediano; el tipo holandés (también llamado europeo), cuyos frutos son largos, con cáscara delgada y no poseen semillas y el tipo mini (también llamado Beit Alpha), cuyos frutos son pequeños, no poseen semillas, su cáscara es delgada y lisa y son menos susceptibles a los daños poscosecha que el tipo holandés (Soleimani, y otros, 2009). Los pepinos Beit Alpha producen 2 o 3 veces más frutos comercializables por planta en comparación con los pepinos holandeses (Soleimani y otros, 2009).

El coeficiente de correlación es una herramienta estadística que generalmente muestra relaciones entre caracteres independientes y el grado de relación lineal entre esas características. En la investigación en fitomejoramiento, por lo general, se toman medidas de varios caracteres o variables a partir de la misma planta. Para determinar el grado y la dirección de las relaciones lineales que corresponden a esas mediciones realizadas, usualmente los investigadores utilizan el análisis de correlación simple (Cancaya, Balkaya, \& Karaagac, 2010; Ene, Ogbonna, Agbo, \& Chukwudi, 2016).

El establecimiento de correlaciones entre caracteres y el grado de su influencia recíproca resultan útiles en los procesos de selección genética. El conocimiento de las correlaciones ofrece la posibilidad de evaluar los caracteres complejos mediante la evaluación de los caracteres menos complejos (Madosa, Ciulca, Velicevici, Avadanei, Sasu, Cioroga, \& Friskan, 2008).

El objetivo de esta investigación fue establecer correlaciones de Pearson entre siete variables cuantitativas para el cultivo de pepino bajo ambiente protegido en Alajuela, Costa Rica.

\section{Materiales y métodos}

Se evaluaron 14 genotipos híbridos de pepino (Cucumis satious L.) partenocárpico (tabla 1); el 
Tabla 1

Genotipos de pepino utilizados en el ensayo

\begin{tabular}{cll}
\hline Tipo de pepino & Genotipo & Empresa \\
\hline & Arioso & Known You Seed \\
Largo & Cumlaude & Dreamliner \\
& Kalunga & Rijk Zwaan \\
& Paisaje & Enza Zaden \\
& Roxinante & Rijk Zwaan \\
& Corinto & Enza Zaden \\
Mediano & Macario & Enza Zaden \\
$(\mathrm{n}=5)$ & Modan & Enza Zaden \\
& Paraíso & Rijk Zwaan \\
& Primavera & Enza Zaden \\
Pequeño & Katrina & Enza Zaden \\
$(\mathrm{n}=3)$ & $22-20-782$ & Enza Zaden \\
& $22-20-783$ & Pandia Seeds \\
\hline
\end{tabular}

Nota. Chacón-Padilla y Monge-Pérez, 2020, a partir de los genotipos utilizados.

cultivo se realizó en condiciones hidropónicas, en un invernaderoubicadoen la Estación Experimental Agrícola Fabio Baudrit Moreno (EEAFBM), en Barrio San José, Alajuela, Costa Rica, a una altitud de 883 msnm.

El almácigo se sembró el 27 de enero de 2015; se utilizaron bandejas de 98 celdas rellenas con turba como sustrato. El trasplante se realizó el 9 de febrero de 2015, cuando las plántulas tenían una hoja verdadera. El período de cultivo abarcó hasta el 14 de mayo de 2015 (94 días después del trasplante-ddt) para un período de cosecha de 10 semanas.

El cultivo se efectuó en sacos plásticos rellenos con fibra de coco, de $1 \mathrm{~m}$ de largo, $20 \mathrm{~cm}$ de ancho y $15 \mathrm{~cm}$ de altura. La distancia de siembra fue de $25 \mathrm{~cm}$ entre plantas, y de 1,54 m entre hileras, para una densidad de 2,60 plantas/m2. Las plantas se manejaron a un solo tallo. Se eliminaron todos los tallos secundarios. Se retiraron los primeros cuatro frutos de cada planta para lograr una cosecha más uniforme. Se recopilaron datos de temperatura, humedad relativa y radiación PAR dentro del invernadero por medio de sensores electrónicos especializados.

Se clasificó la cosecha según categorías de calidad (tabla 2). Se consideró como rendimiento comercial la suma de las categorías de primera y segunda calidad y como rendimiento total la suma de las tres categorías de calidad.

Se evaluaron las siguientes variables:

1. Longitud del fruto $(\mathrm{cm})$ : se midió esta característica a 20 frutos de cada categoría de calidad y se obtuvo el promedio.

2. Diámetro del fruto $(\mathrm{mm})$ : se midió esta característica en la parte media de 20 frutos de cada categoría de calidad y se obtuvo el promedio. 
Tabla 2

Parámetros de calidad de pepino utilizados en el ensayo

\begin{tabular}{llll}
\hline \multirow{2}{*}{ Parámetro } & \multicolumn{3}{c}{ Categoría de calidad } \\
\cline { 2 - 4 } & Primera & Segunda & Rechazo \\
\hline Deformación de frutos & Ausente & Leve & Severa \\
Daños en la cáscara del fruto & Ausente & Menor o igual a $1 \mathrm{~cm} 2$ & Mayor a 1 cm2 \\
\hline
\end{tabular}

Nota. Elaboración propia basado en Chacón-Padilla y Monge-Pérez, 2020.

3. Número de frutos por planta: se contabilizó el número total de frutos por parcela y se dividió entre el número de plantas de la parcela.

4. Peso del fruto (g): se midió el peso total de la producción en cada parcela y se dividió entre el número total de frutos por parcela.

5. Rendimiento total $(\mathrm{kg} / \mathrm{m} 2)$ : se calculó a partir del rendimiento por planta y de la densidad de siembra.

6. Rendimiento comercial $(\mathrm{kg} / \mathrm{m} 2)$ : se calculó a partir del rendimiento por planta y de la densidad de siembra. Se consideraron los frutos de primera y de segunda calidad.

7. Porcentaje de sólidos solubles totales ( ${ }^{\circ}$ Brix): se evaluó esta característica en la parte media (pulpa y placenta) de 20 frutos de cada categoría de calidad y se obtuvo el promedio.

El peso de los frutos se determinó por medio de una balanza electrónica marca Ocony, modelo UWE HGM, con una capacidad de $20000 \pm 1 \mathrm{~g}$. Para obtener la longitud del fruto, se utilizó una cinta métrica marca Assist, modelo 32G-8025, con una capacidad de $800,0 \pm 0,1 \mathrm{~cm}$. El diámetro de los frutos se determinó con un calibrador digital marca Mitutoyo, modelo CD, y una capacidad de 15,00 \pm $0,01 \mathrm{~cm}$. El porcentaje de sólidos solubles totales se midió con un refractómetro manual marca Boeco con una capacidad de $32,0 \pm 0,2^{\circ}$ Brix.

Se utilizó un diseño experimental irrestricto al azar con cuatro repeticiones por genotipo. La parcela consistió de ocho plantas (dos sacos), y todas las plantas fueron evaluadas. Para las siete variables evaluadas, se obtuvo el coeficiente de correlación de Pearson (r) entre ellas y para aquellas combinaciones de variables en que se obtuvo una alta correlación $(r \geq 0,66)$ y significancia estadística $(\mathrm{p} \leq 0,05)$, se calculó la regresión lineal con su respectiva ecuación y coeficiente de determinación (R2). Las correlaciones se obtuvieron tanto entre el total de los 14 genotipos evaluados, según el tipo de pepino (largo, mediano y pequeño).

\section{Resultados y discusión}

Durante el desarrollo del ensayo, la temperatura dentro del invernadero varió entre 14 y $41^{\circ} \mathrm{C}$, la humedad relativa entre 18 y $95 \%$ y la radiación PAR entre y 250 y 2250 W/m2.

En la tabla 3 se presentan los estimadores estadísticos principales de las variables evaluadas.

En los cuadros 4 al 13, se presentan las correlaciones de Pearson para las combinaciones de las variables evaluadas en que el resultado fue alto $(r \geq 0,66)$ y estadísticamente significativo $(p \leq 0,05)$ y en las figuras 1 al 15, las regresiones lineales de dichas combinaciones. No se ofrecen losofrecen los resultados para las combinaciones de variables en que la correlación entre el total de 14 genotipos, o entre los grupos de cada tipo de pepino, resultó ser no significativa o fue menor a 0,66 . 
Tabla 3

Estimadores estadísticos principales de las variables evaluadas

\begin{tabular}{llll}
\hline \multirow{2}{*}{ Variable } & \multicolumn{3}{c}{ Estimador estadístico } \\
\cline { 2 - 4 } & Promedio & Valor mínimo & Valor máximo \\
\hline Longitud del fruto $(\mathrm{cm})$ & 26,42 & 17,24 & 36,19 \\
Diámetro del fruto $(\mathrm{mm})$ & 47,77 & 41,94 & 52,99 \\
Porcentaje de sólidos & $30 l u b l e s$ & 4,17 \\
totales ( ${ }^{\circ}$ Brix) & 3,45 & 2,84 & 501,92 \\
Peso del fruto $(\mathrm{g})$ & 374,26 & 216,15 & 46,99 \\
Número de frutos por planta & 24,36 & 12,38 & 30,58 \\
Rendimiento total $(\mathrm{kg} / \mathrm{m} 2)$ & 21,80 & 14,42 & 23,73 \\
Rendimiento comercial $(\mathrm{kg} / \mathrm{m} 2)$ & 17,23 & 11,64 & \multicolumn{3}{c}{} \\
\hline
\end{tabular}

Nota. Chacón-Padilla y Monge-Pérez, 2020, a partir de los datos obtenidos. laboración propia

Con respecto a la correlación entre la longitud y el peso del fruto, el coeficiente obtenido fue alto, positivo y altamente significativo, pero únicamente para el total de 14 genotipos (figura 1), mientras que fue no significativo para cada grupo según el tipo de pepino (tabla 4). Esto indica que, dentro de cada tipo de pepino, no hay una relación significativa entre estas variables.

Otros autores encontraron también una correlación alta, positiva y altamente significativa entre ambas variables $(\mathrm{r}=0,61-0,87)$ (Deepa, $\mathrm{y}$ otros, 2018; Ullah, Hasan, Chowdhury, Saki, \& Rahman, 2012; Pal, y otros, 2017; Hanchinamani, 2006; Veena, Sidhu, Pitchaimuthu, \& Souravi, 2013). En otros casos, la correlación obtenida fue baja, positiva y significativa $(\mathrm{r}=0,21-0,24)$ (Golabadi, Golkar, \& Eghtedary, 2015; Nwofia, y otros, 2015). Otros investigadores hallaron una correlación no significativa entre estas variables (Ahirwar, Singh, \& Kushwaha, 2017; Kumari, Singh, Moharana, Kumar, \& Kumar, 2018; Sharma, y otros, 2018).
En cuanto a la correlación entre el diámetro $y$ el peso del fruto, el coeficiente obtenido fue alto, positivo y altamente significativo, pero únicamente para el total de 14 genotipos (figura 2), mientras que fue positivo y significativo para el pepino pequeño y fue no significativo para los pepinos tipo largo y mediano (tabla 5). Por lo tanto, en fitomejoramiento de pepino tipo pequeño, se puede aprovechar la relación directamente proporcional y significativa entre estas variables.

Otros investigadores hallaron también una correlación alta, positiva y altamente significativa entre ambas variables $(r=0,62-0,74)$ (Kumari, y otros, 2018; Ullah, y otros, 2012; Pal, y otros, 2017; Deepa, y otros, 2018). En otros estudios, se halló una correlación positiva, y significativa o altamente significativa, aunque baja, entre estas variables $(r=0,21-0,48)$ (Hanchinamani, 2006; Veena, y otros, 2013; Golabadi, y otros, 2015; Sharma, y otros, 2018) y otros investigadores encontraron una correlación no significativa (Ahirwar, y otros, 2017; Nwofia, y otros, 2015). 
Chacón-Padilla \& Monge-Pérez

Tabla 4

Coeficientes de correlación de Pearson entre longitud del fruto y peso del fruto

\begin{tabular}{lccc}
\hline Genotipos & $\begin{array}{c}\text { Coeficiente de co- } \\
\text { rrelación (r) }\end{array}$ & Probabilidad (p) & $\begin{array}{c}\text { Coeficiente de deter- } \\
\text { minación de la re- } \\
\text { gresión lineal (R2) }\end{array}$ \\
\cline { 2 - 4 } Total $(\mathrm{n}=14)$ & 0,92 & $* *$ & 0,84 \\
\hline Largo $(\mathrm{n}=6)$ & 0,02 & $\mathrm{~ns}$ & \\
Mediano $(\mathrm{n}=5)$ & $-0,14$ & $\mathrm{~ns}$ \\
Pequeño $(\mathrm{n}=3)$ & $-0,10$ & $\mathrm{~ns}$ & \\
\hline
\end{tabular}

Nota. Chacón-Padilla y Monge-Pérez, 2020, a partir de los datos obtenidos. Simbología: ns = no significativo; ${ }^{*}=$ significativa $(p \leq 0,05) ;{ }^{* *}=$ altamente significativa $(p \leq 0,01)$.

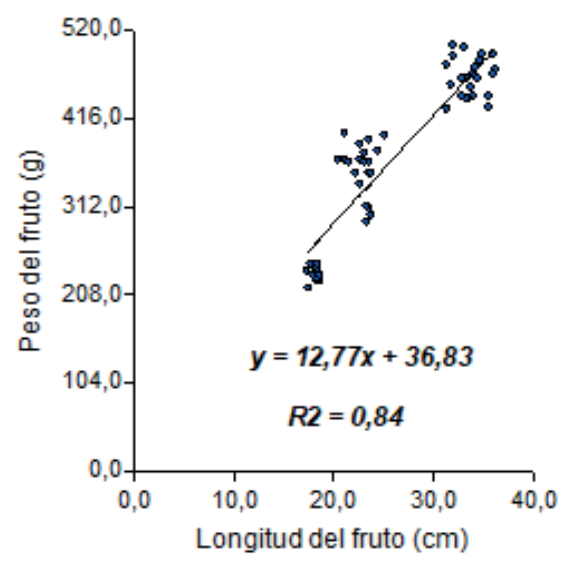

Figura 1. Regresión lineal entre longitud del fruto y peso del fruto para el total de genotipos evaluados $(\mathrm{n}=14)$. Nota. Chacón-Padilla y Monge-Pérez, 2020, a partir de los datos obtenidos.

Tabla 5

Coeficientes de correlación de Pearson entre diámetro del fruto y peso del fruto

\begin{tabular}{lccc}
\hline Genotipos & $\begin{array}{c}\text { Coeficiente de co- } \\
\text { rrelación (r) }\end{array}$ & Probabilidad (p) & $\begin{array}{c}\text { Coeficiente de deter- } \\
\text { minación de la re- } \\
\text { gresión lineal (R2) }\end{array}$ \\
\cline { 2 - 4 } Total $(\mathrm{n}=14)$ & 0,66 & $* *$ & 0,44 \\
\hline Largo $(\mathrm{n}=6)$ & 0,25 & $\mathrm{~ns}$ & \\
Mediano $(\mathrm{n}=5)$ & 0,26 & $\mathrm{~ns}$ & \\
Pequeño $(\mathrm{n}=3)$ & 0,60 & $*$ \\
\hline
\end{tabular}

Nota. Elaboración propia, a partir de los datos obtenidos. Simbología: ns = no significativo; ${ }^{*}=$ significativa $(p \leq 0,05) ; * *=$ altamente significativa $(p \leq 0,01)$. 


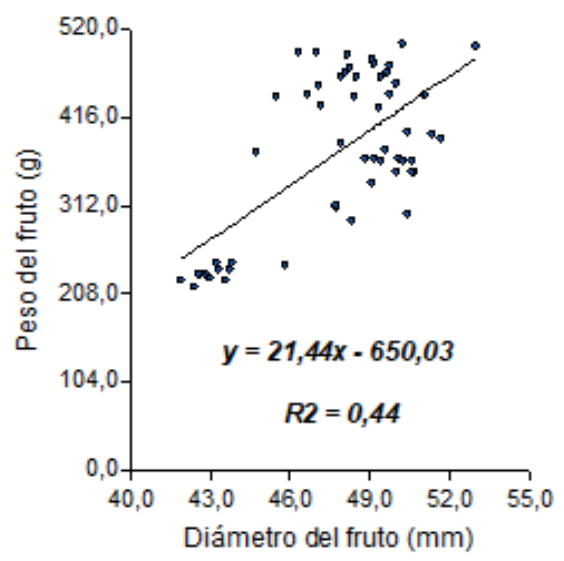

Figura 2. Regresión lineal entre diámetro del fruto y peso del fruto para el total de genotipos evaluados $(\mathrm{n}=14)$. Nota. Chacón-Padilla y Monge-Pérez, 2020, a partir de los datos obtenidos. .

Se obtuvo una correlación alta, negativa y altamente significativa entre el peso del fruto y el número total de frutos por planta, pero únicamente para el total de 14 genotipos (figura $3)$, mientras que fue no significativa para cada grupo según el tipo de pepino (tabla 6).

En forma contraria a lo obtenido en el presente ensayo, en otros estudios, se halló una correlación positiva y altamente significativa entre ambas variables $(r=0,55-0,63)$ (Hanchinamani, 2006; Kumari, y otros, 2018); es decir, que, a mayor peso del fruto, también se obtuvo un mayor número total de frutos por planta. Estos disímiles resultados se pueden deber a las diferencias en los materiales genéticos evaluados y en las condiciones climáticas.

Otros autores, al igual que en el presente ensayo, hallaron una correlación negativa $\mathrm{y}$ significativa entre estas variables $(r=-0,29)$ (Pal, y otros, 2017), mientras que en otros trabajos se encontró una correlación no significativa (Deepa, y otros, 2018; Veena, y otros, 2013; Ullah y otros, 2012; Golabadi, y otros, 2015; Ahirwar y otros, 2017; Nwofia, y otros, 2015; Sharma y otros, 2018).

Se halló una correlación alta, negativa y altamente significativa entre el diámetro del fruto y el número total de frutos por planta, pero únicamente para el total de 14 genotipos (figura 4), mientras que fue negativa y altamente significativa, pero no muy alta, para el pepino largo, y no significativa para los pepinos tipo mediano y pequeño (tabla 7). Por lo tanto, en el fitomejoramiento de pepino largo, es importante tomar en cuenta la relación entre el diámetro del fruto y el número total de frutos por planta, pues la selección de frutos de menor diámetro puede resultar en una mayor productividad de frutos.

En forma contraria a lo hallado en la presente investigación, otros investigadores encontraron una correlación alta, positiva y altamente significativa entre ambas variables $(r=0,86$ 0,88) (Ene y otros, 2016), es decir, que se obtuvo un mayor número de frutos por planta conforme aumentó el diámetro del fruto. En otros estudios, se evidenció una correlación positiva $\mathrm{y}$ altamente significativa, aunque no tan alta ( $\mathrm{r}$ = 0,52-0,54) (Hanchinamani, 2006; Kumari y otros, 2018).

En forma similar a lo obtenido en el presente ensayo, otros investigadores hallaron una correlación negativa y significativa o altamente significativa, aunque no muy alta, entre estas 


\section{Chacón-Padilla \& Monge-Pérez}

Tabla 6

Coeficientes de correlación de Pearson entre peso del fruto y número total de frutos por planta

\begin{tabular}{lccc}
\hline Genotipos & $\begin{array}{c}\text { Coeficiente de co- } \\
\text { rrelación }(\mathrm{r})\end{array}$ & Probabilidad $(\mathrm{p})$ & $\begin{array}{c}\text { Coeficiente de deter- } \\
\text { minación de la re- } \\
\text { gresión lineal (R2) }\end{array}$ \\
\cline { 2 - 4 } Total $(\mathrm{n}=14)$ & $-0,84$ & $* *$ & 0,70 \\
\hline Largo $(\mathrm{n}=6)$ & 0,08 & $\mathrm{~ns}$ & \\
Mediano $(\mathrm{n}=5)$ & $-0,16$ & $\mathrm{~ns}$ & \\
Pequeño $(\mathrm{n}=3)$ & $-0,07$ & $\mathrm{~ns}$ & \\
\hline
\end{tabular}

Nota. Chacón-Padilla y Monge-Pérez, 2020, a partir de los datos obtenidos. Simbología: ns = no significativo; ${ }^{*}=$ significativa $(p \leq 0,05) ;{ }^{*}=$ altamente significativa $(p \leq 0,01)$.

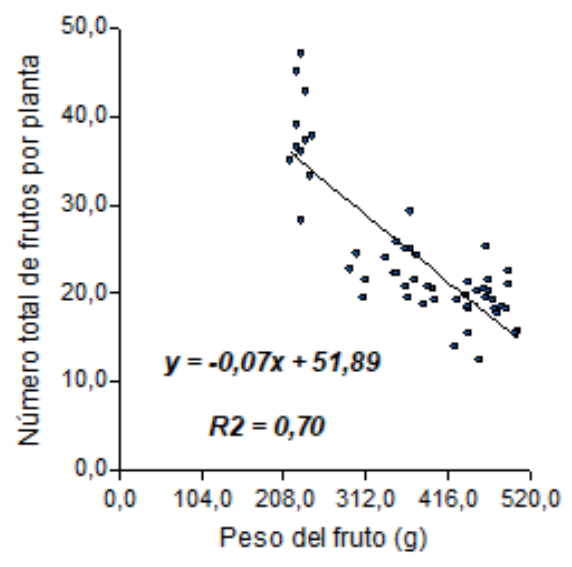

Figura 3. Regresión lineal entre peso del fruto y número total de frutos por planta para el total de genotipos evaluados $(\mathrm{n}=14)$.

Nota. Chacón-Padilla y Monge-Pérez, 2020, a partir de los datos obtenidos.

Tabla 7

Coeficientes de correlación de Pearson entre diámetro del fruto y número total de frutos por planta

\begin{tabular}{lccc}
\hline Genotipos & $\begin{array}{c}\text { Coeficiente de co- } \\
\text { rrelación }(\mathrm{r})\end{array}$ & Probabilidad $(\mathrm{p})$ & $\begin{array}{c}\text { Coeficiente de deter- } \\
\text { minación de la re- } \\
\text { gresión lineal (R2) }\end{array}$ \\
\cline { 2 - 4 } Total $(\mathrm{n}=14)$ & $-0,77$ & $* *$ & 0,59 \\
\hline Largo $(\mathrm{n}=6)$ & $-0,53$ & $* *$ & \\
Mediano $(\mathrm{n}=5)$ & 0,12 & $\mathrm{~ns}$ & \\
Pequeño $(\mathrm{n}=3)$ & $-0,13$ & $\mathrm{~ns}$ & \\
\hline
\end{tabular}

Nota. Chacón-Padilla y Monge-Pérez, 2020, a partir de los datos obtenidos. Simbología: ns = no significativo; ${ }^{*}=$ significativa $(p \leq 0,05) ;{ }^{* *}=$ altamente significativa $(p \leq 0,01)$. 


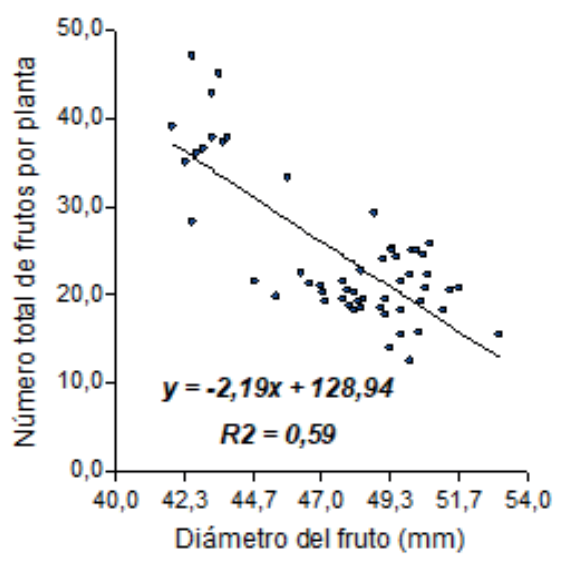

Figura 4. Regresión lineal entre diámetro del fruto y número total de frutos por planta para el total de genotipos evaluados $(\mathrm{n}=14)$.

Nota. Chacón-Padilla y Monge-Pérez, 2020, a partir de los datos obtenidos.

variables ( $\mathrm{r}=-0,26$ a $-0,33)$ (Veena, y otros, 2013; Golabadi, y otros, 2015; Sharma, y otros, 2018); $\mathrm{y}$ otros autores encontraron una correlación no significativa (Deepa, y otros, 2018; Shet, Shantappa, Ashok, \& Gurumurthy, 2018; Pal, y otros, 2017; Ahirwar, y otros, 2017; Ullah, y otros, 2012; Soleimani, y otros, 2009; Nwofia, y otros, 2015).

Con respecto a la correlación entre el diámetro del fruto y el porcentaje de sólidos solubles totales, el coeficiente obtenido fue alto, positivo y altamente significativo, pero únicamente para el total de 14 genotipos (figura 5); $y$, de forma contraria, fue alto, negativo y altamente significativo para el pepino tipo pequeño (figura 6), mientras que fue no significativo para los pepinos tipo largo y mediano (tabla 8). Estos resultados indican que, en el caso de los genotipos de pepino tipo pequeño, a mayor diámetro de fruto, se obtuvo un menor porcentaje de sólidos solubles totales. Por lo tanto, es una relación significativa por tomar en cuenta en la selección genética.

De forma similar a lo obtenido en el presente trabajo, unos investigadores encontraron una correlación baja, negativa y altamente significativa entre ambas variables $(r=-0,37)$
(Pal, y otros, 2017) y otros autores evidenciaron una correlación no significativa (Sharma, y otros, 2018).

En cuanto a la correlación entre el número total de frutos por planta y el porcentaje de sólidos solubles totales, el coeficiente obtenido fue alto, negativo y altamente significativo, pero únicamente para el total de 14 genotipos (figura 7), mientras que fue no significativo para cada grupo según el tipo de pepino (tabla 9).

Varios autores identificaron una correlación no significativa entre ambas variables (Sharma, y otros, 2018; Pal, y otros, 2017).

Se encontró una correlación alta, negativa y altamente significativa entre la longitud del fruto y el número total de frutos por planta, pero únicamente para el total de 14 genotipos (figura $8)$, pero esta correlación fue positiva, altamente significativa, pero no muy alta, para el pepino largo y fue no significativa para los pepinos tipo mediano y pequeño (tabla 10). Por lo tanto, la relación entre estas variables es significativa en fitomejoramiento de pepino tipo largo, pues la selección de frutos de mayor longitud está asociada a la obtención de un mayor número total de frutos por planta. 
Chacón-Padilla \& Monge-Pérez

Tabla 8

Coeficientes de correlación de Pearson entre diámetro del fruto y porcentaje de sólidos solubles totales

\begin{tabular}{lccc}
\hline Genotipos & $\begin{array}{c}\text { Coeficiente de co- } \\
\text { rrelación }(\mathrm{r})\end{array}$ & Probabilidad (p) & $\begin{array}{c}\text { Coeficiente de deter- } \\
\text { minación de la re- } \\
\text { gresión lineal (R2) }\end{array}$ \\
\cline { 2 - 4 } Total $(\mathrm{n}=14)$ & 0,69 & $* *$ & 0,47 \\
\hline Largo $(\mathrm{n}=6)$ & 0,13 & $\mathrm{~ns}$ & \\
Mediano $(\mathrm{n}=5)$ & 0,09 & $\mathrm{~ns}$ & 0,47 \\
Pequeño $(\mathrm{n}=3)$ & $-0,69$ & $* *$ & \\
\hline
\end{tabular}

Nota. Chacón-Padilla y Monge-Pérez, 2020, a partir de los datos obtenidos. Simbología: ns = no significativo; ${ }^{*}=$ significativa $(p \leq 0,05) ;{ }^{* *}=$ altamente significativa $(p \leq 0,01)$.

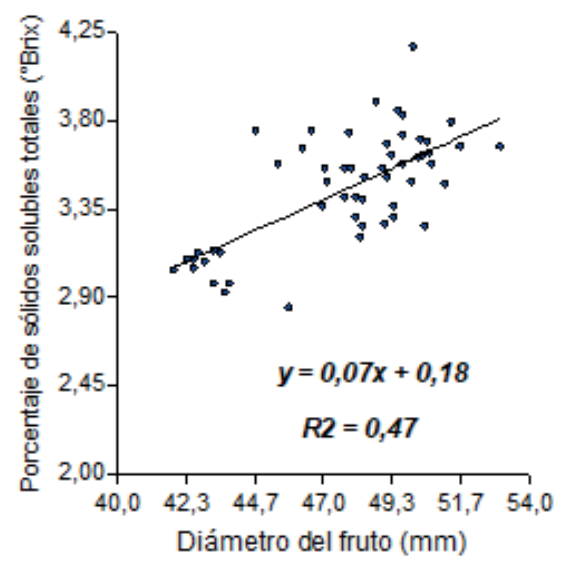

Figura 5. Regresión lineal entre diámetro del fruto y porcentaje de sólidos solubles totales para el total de genotipos evaluados $(\mathrm{n}=14)$.

Nota. Chacón-Padilla y Monge-Pérez, 2020, a partir de los datos obtenidos.

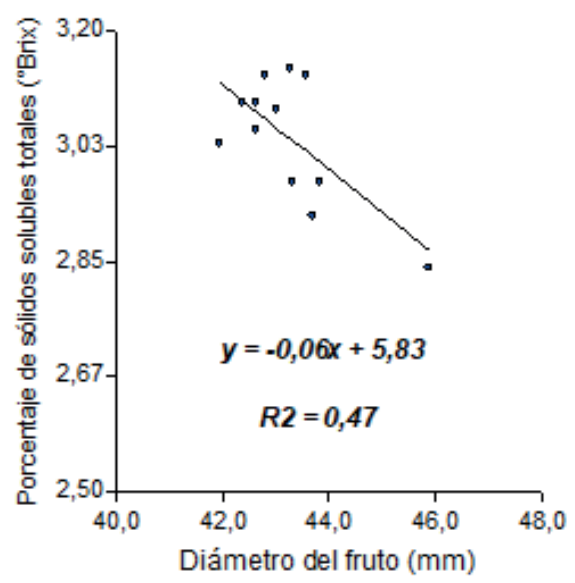

Figura 6. Regresión lineal entre diámetro del fruto y porcentaje de sólidos solubles totales para los genotipos de pepino pequeño $(n=3)$.

Nota. Chacón-Padilla y Monge-Pérez, 2020, a partir de los datos obtenidos. 
Producción de pepino (Cucumis sativus L.) bajo invernadero

Tabla 9

Coeficientes de correlación de Pearson entre diámetro del fruto y porcentaje de sólidos solubles totales

\begin{tabular}{lccc}
\hline Genotipos & $\begin{array}{c}\text { Coeficiente de co- } \\
\text { rrelación (r) }\end{array}$ & Probabilidad (p) & $\begin{array}{c}\text { Coeficiente de deter- } \\
\text { minación de la re- } \\
\text { gresión lineal (R2) }\end{array}$ \\
\cline { 2 - 4 } Total $(\mathrm{n}=14)$ & $-0,67$ & $* *$ & 0,45 \\
\hline Largo $(\mathrm{n}=6)$ & $-0,27$ & $\mathrm{~ns}$ & \\
Mediano $(\mathrm{n}=5)$ & 0,13 & $\mathrm{~ns}$ & \\
Pequeño $(\mathrm{n}=3)$ & 0,24 & $\mathrm{~ns}$ & \\
\hline
\end{tabular}

Nota. Chacón-Padilla y Monge-Pérez, 2020, a partir de los datos obtenidos. Simbología: ns = no significativo; ${ }^{*}=$ significativa $(p \leq 0,05) ;{ }^{* *}=$ altamente significativa $(p \leq 0,01)$.

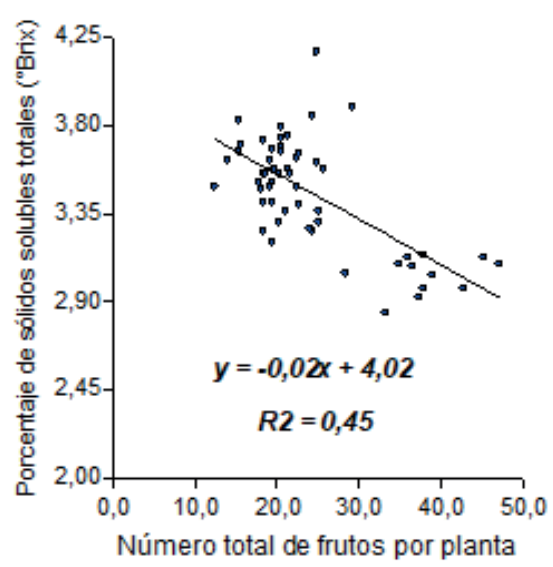

Figura 7. Regresión lineal entre número total de frutos por planta y porcentaje de sólidos solubles totales para el total de genotipos evaluados $(n=14)$.

Nota. Chacón-Padilla y Monge-Pérez, 2020, a partir de los datos obtenidos.

Tabla 10

Coeficientes de correlación de Pearson entre longitud del fruto y número total de frutos por planta

\begin{tabular}{lccc}
\hline Genotipos & $\begin{array}{c}\text { Coeficiente de co- } \\
\text { rrelación (r) }\end{array}$ & Probabilidad (p) & $\begin{array}{c}\text { Coeficiente de deter- } \\
\text { minación de la re- } \\
\text { gresión lineal (R2) }\end{array}$ \\
\cline { 2 - 4 } Total $(\mathrm{n}=14)$ & $-0,72$ & $* *$ & 0,52 \\
\hline Largo $(\mathrm{n}=6)$ & 0,50 & $* *$ & \\
Mediano $(\mathrm{n}=5)$ & $-0,01$ & $\mathrm{~ns}$ & \\
Pequeño $(\mathrm{n}=3)$ & $-0,26$ & $\mathrm{~ns}$ & \\
\hline
\end{tabular}

Nota. Chacón-Padilla y Monge-Pérez, 2020, a partir de los datos obtenidos. Simbología: ns = no significativo; ${ }^{*}=$ significativa $(p \leq 0,05) ;{ }^{* *}=$ altamente significativa $(p \leq 0,01)$. 


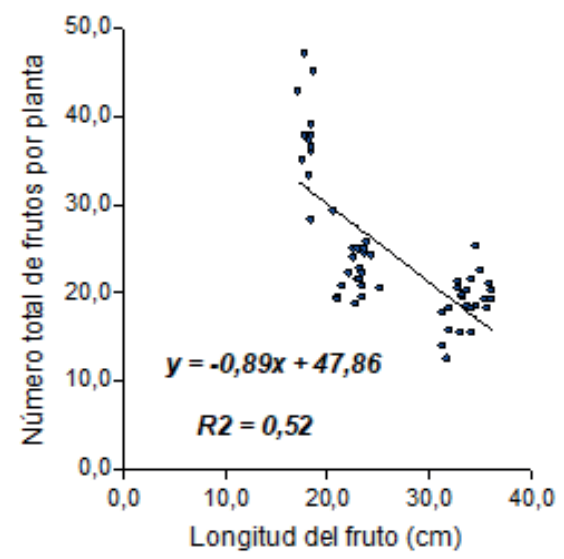

Figura 8. Regresión lineal entre longitud del fruto y número total de frutos por planta para el total de genotipos evaluados $(n=14)$.

Nota. Chacón-Padilla y Monge-Pérez, 2020, a partir de los datos obtenidos.

Al comparar los diferentes tipos de pepino, en el caso del pepino pequeño, al producir frutos de menor tamaño en comparación a los otros tipos, esto le permite a la planta desarrollar en forma adecuada un mayor número de frutos, a partir de los fotoasimilados producidos, mientras que al aumentar la longitud del fruto la planta produce una menor cantidad de ellos. Además, en las plantas de pepino largo se observó que solo se produce un fruto por nudo, mientras que en el pepino pequeño cada nudo puede llegar a producir más de 15 flores y se llegan a cosechar varios frutos por nudo.

$\mathrm{Al}$ igual que lo obtenido en el presente ensayo, para el total de genotipos, otros autores también encontraron una correlación negativa y altamente significativa, aunque no muy alta, entre ambas variables $(\mathrm{r}=-0,56)$ (Ullah, y otros, 2012).

Al igual que lo investigado en el presente estudio con los pepinos tipo largo, en otros ensayos se detectó una correlación alta, positiva $\mathrm{y}$ altamente significativa entre estas variables ( $\mathrm{r}$ $=0,78-0,91)$ (Hanchinamani, 2006; Ene, y otros, 2016). Otros investigadores determinaron una correlación positiva y significativa, aunque no muy alta $(r=0,50)$ (Afangideh \& Uyoh, 2007) y otros autores identificaron una correlación no significativa (Ahirwar, y otros, 2017; Kumari, y otros, 2018; Soleimani, y otros, 2009; Nwofia, y otros, 2015; Sharma, y otros, 2018; Deepa, y otros, 2018; Shet, y otros, 2018; Veena, y otros, 2013; Golabadi, y otros, 2015; Pal, y otros, 2017). Estos resultados tan disímiles, se pueden deber a los diferentes materiales genéticos involucrados en cada estudio, así como a las condiciones climáticas prevalecientes en cada zona agroecológica y a las particulares de manejo del cultivo.

Se obtuvo una correlación alta, positiva y altamente significativa entre el rendimiento total y el rendimiento comercial para el total de 14 genotipos (figura 9), al igual que para cada grupo según el tipo de pepino (tabla 11). Esto evidencia la gran relevancia de la relación entre ambas variables para todos los tipos de pepino.

$\mathrm{Al}$ igual que los resultados obtenidos en el presente trabajo, en otro estudio se halló también una correlación muy alta, positiva y altamente significativa entre ambas variables $(r=0,99)$ (Hanchinamani, 2006).

Con respecto a la correlación entre el número total de frutos por planta y el rendimiento total, el coeficiente obtenido fue bajo y positivo, aunque altamente significativo, para el total 
Tabla 11

Coeficientes de correlación de Pearson entre rendimiento total y rendimiento comercial

\begin{tabular}{lccc}
\hline Genotipos & $\begin{array}{c}\text { Coeficiente de co- } \\
\text { rrelación }(\mathrm{r})\end{array}$ & Probabilidad (p) & $\begin{array}{c}\text { Coeficiente de deter- } \\
\text { minación de la re- } \\
\text { gresión lineal (R2) }\end{array}$ \\
\cline { 2 - 4 } Total $(\mathrm{n}=14)$ & 0,81 & $* *$ & 0,66 \\
\hline Largo $(\mathrm{n}=6)$ & 0,89 & $* *$ & 0,79 \\
Mediano $(\mathrm{n}=5)$ & 0,95 & $* *$ & 0,90 \\
Pequeño $(\mathrm{n}=3)$ & 0,96 & $* *$ & 0,92 \\
\hline
\end{tabular}

Nota. Chacón-Padilla y Monge-Pérez, 2020, a partir de los datos obtenidos. Simbología: ns = no significativo; ${ }^{*}=$ significativa $(p \leq 0,05) ;{ }^{* *}=$ altamente significativa $(p \leq 0,01)$.

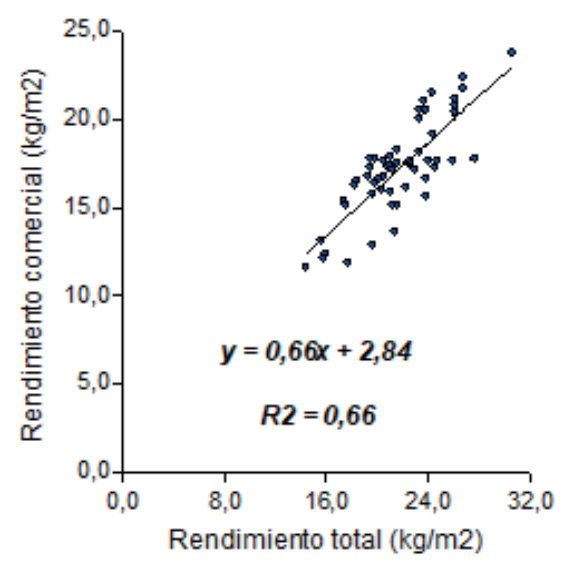

Figura 9. Regresión lineal entre el rendimiento total y el rendimiento comercial para el total de genotipos evaluados $(\mathrm{n}=14)$.

Nota. Chacón-Padilla y Monge-Pérez, 2020, a partir de los datos obtenidos.

de 14 genotipos (tabla 12); sin embargo, sí fue alto, positivo y altamente significativo para el pepino largo (figura 10), el mediano (figura 11), y el pequeño (figura 12). Estos resultados evidencian la gran significancia de la relación entre estas dos variables, para cada tipo de pepino, por lo que el número total de frutos por planta se destaca como una variable esencial en la selección genética y el fitomejoramiento del pepino.
Al igual que los resultados del presente trabajo, también, en otros estudios, se identificó una correlación alta, positiva y altamente significativa entre ambas variables $(r=0,66-$ 0,93) (Hanchinamani, 2006; Ahirwar, y otros, 2017; Shet y otros, 2018; Kumari, y otros, 2018; Afangideh \& Uyoh, 2007; Ene y otros, 2016; Golabadi y otros, 2015; Soleimani, y otros, 2009; Pal, y otros, 2017; Sharma y otros, 2018). 
Tabla 12

Coeficientes de correlación de Pearson entre número total de frutos por planta y rendimiento total

\begin{tabular}{lccc}
\hline Genotipos & $\begin{array}{c}\text { Coeficiente de co- } \\
\text { rrelación (r) }\end{array}$ & Probabilidad (p) & $\begin{array}{c}\text { Coeficiente de deter- } \\
\text { minación de la re- } \\
\text { gresión lineal (R2) }\end{array}$ \\
\cline { 2 - 4 } Total $(\mathrm{n}=14)$ & 0,39 & $* *$ & \\
\hline Largo $(\mathrm{n}=6)$ & 0,97 & $* *$ & 0,93 \\
Mediano $(\mathrm{n}=5)$ & 0,80 & $* *$ & 0,64 \\
Pequeño $(\mathrm{n}=3)$ & 0,95 & $* *$ & 0,89 \\
\hline
\end{tabular}

Nota. Chacón-Padilla y Monge-Pérez, 2020, a partir de los datos obtenidos. Simbología: ns = no significativo; ${ }^{*}=$ significativa $(p \leq 0,05) ;{ }^{* *}=$ altamente significativa $(p \leq 0,01)$.

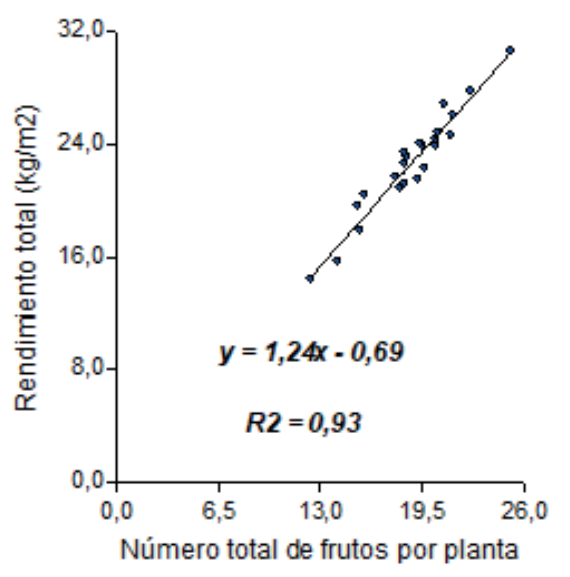

Figura 10. Regresión lineal entre número total de frutos por planta y rendimiento total para los genotipos de pepino largo $(\mathrm{n}=6)$.

Nota. Chacón-Padilla y Monge-Pérez, 2020, a partir de los datos obtenidos. 


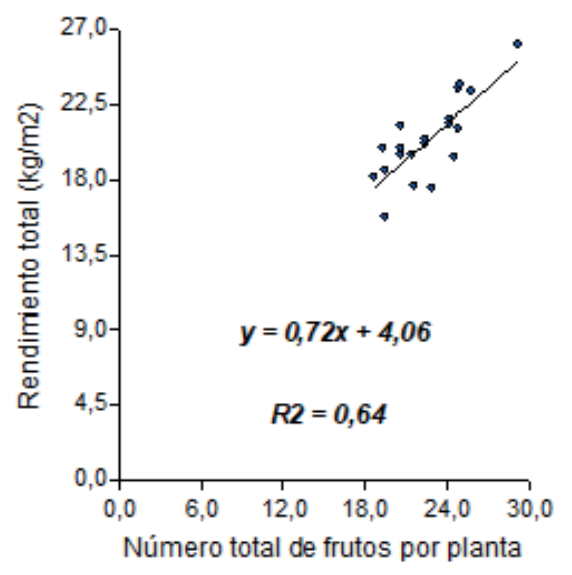

Figura 11. Regresión lineal entre número total de frutos por planta y rendimiento total para los genotipos de pepino mediano $(\mathrm{n}=5)$.

Nota. Chacón-Padilla y Monge-Pérez, 2020, a partir de los datos obtenidos.

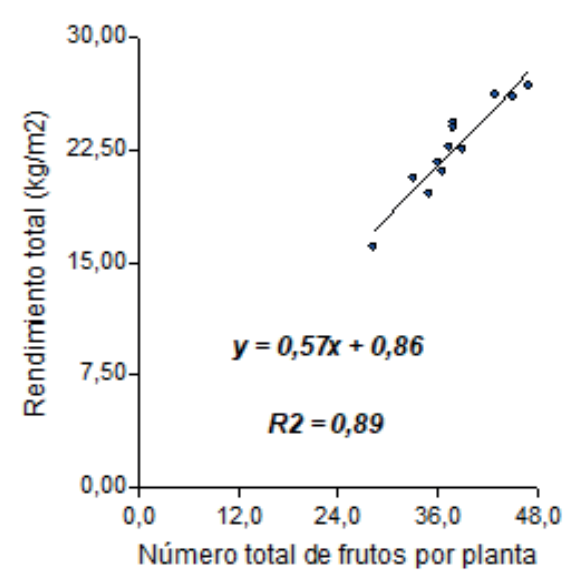

Figura 12. Regresión lineal entre número total de frutos por planta y rendimiento total para los genotipos de pepino pequeño $(\mathrm{n}=3)$.

Nota. Chacón-Padilla y Monge-Pérez, 2020, a partir de los datos obtenidos.

Otros autores encontraron una correlación baja, positiva, y significativa o altamente significativa entre estas variables $(\mathrm{r}=0,37-0,62)$ (Deepa, y otros, 2018; Veena, y otros, 2013; Ullah, y otros, 2012; Nwofia, y otros, 2015).

En cuanto a la correlación entre el número total de frutos por planta y el rendimiento comercial, el coeficiente obtenido fue bajo y positivo, aunque altamente significativo, para el total de 14 genotipos (tabla 13); sin embargo, sí fue alto, positivo y altamente significativo para el pepino largo (figura 13), el mediano (figura 14) y el pequeño (figura 15). Nuevamente, esto enfatiza la importancia de la relación entre ambas variables para los programas de fitomejoramiento en pepino.

De forma similar a lo obtenido en la presente investigación, en otro estudio se halló una correlación alta, positiva y altamente significativa entre ambas variables $(r=0,92)$ (Hanchinamani, 2006). 
Tabla 13

Coeficientes de correlación de Pearson entre número total de frutos por planta y rendimiento comercial

\begin{tabular}{lccc}
\hline Genotipos & $\begin{array}{c}\text { Coeficiente de co- } \\
\text { rrelación }(\mathrm{r})\end{array}$ & Probabilidad (p) & $\begin{array}{c}\text { Coeficiente de deter- } \\
\text { minación de la re- } \\
\text { gresión lineal (R2) }\end{array}$ \\
\cline { 2 - 4 } Total $(\mathrm{n}=14)$ & 0,51 & $* *$ & \\
\hline Largo $(\mathrm{n}=6)$ & 0,82 & $* *$ & 0,67 \\
Mediano $(\mathrm{n}=5)$ & 0,72 & $* *$ & 0,51 \\
Pequeño $(\mathrm{n}=3)$ & 0,84 & $* *$ & 0,71 \\
\hline
\end{tabular}

Nota. Chacón-Padilla y Monge-Pérez, 2020, a partir de los datos obtenidos. Simbología: ns = no significativo; $^{*}=$ significativa $(p \leq 0,05) ;{ }^{* *}=$ altamente significativa $(p \leq 0,01)$.

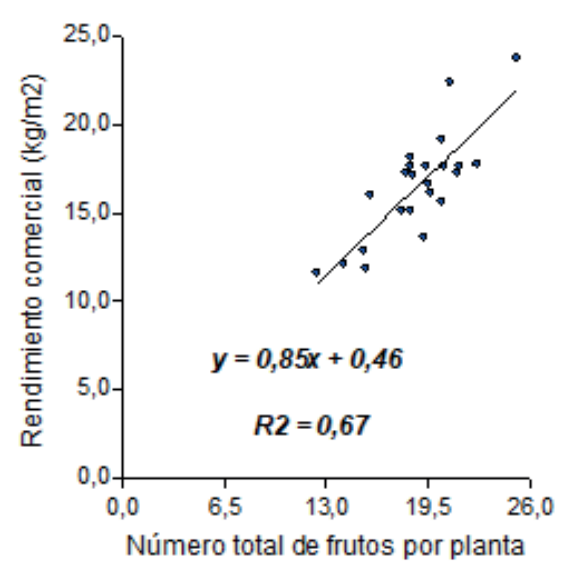

Figura 13. Regresión lineal entre número total de frutos por planta y rendimiento comercial para los genotipos de pepino largo $(n=6)$.

Nota. Chacón-Padilla y Monge-Pérez, 2020, a partir de los datos obtenidos.

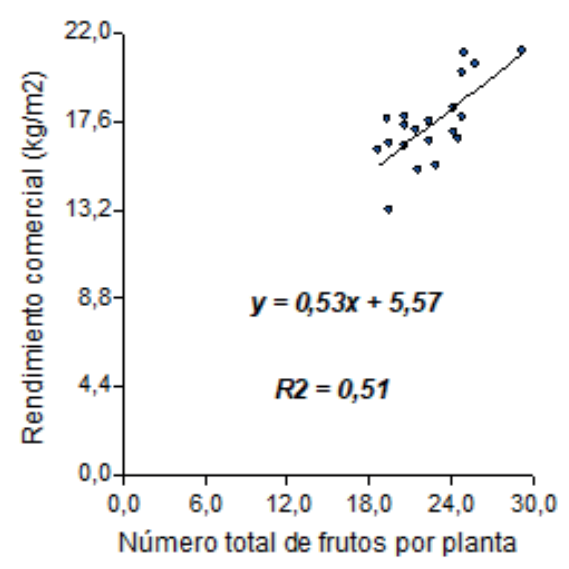

Figura 14. Regresión lineal entre número total de frutos por planta y rendimiento comercial para los genotipos de pepino mediano $(n=5)$.

Nota. Chacón-Padilla y Monge-Pérez, 2020, a partir de los datos obtenidos. 


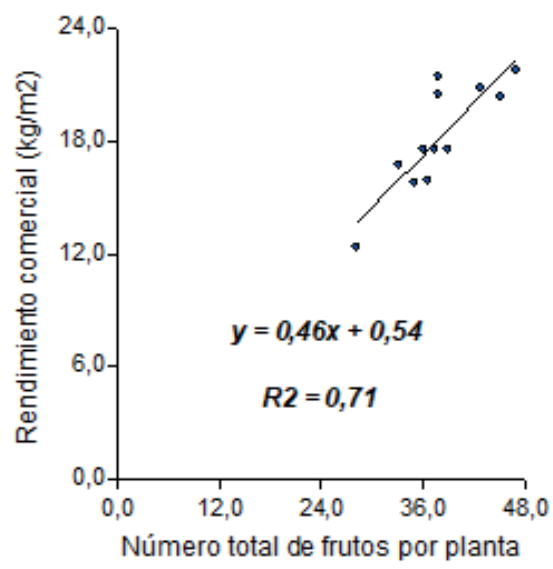

Figura 15. Regresión lineal entre número total de frutos por planta y rendimiento comercial para los genotipos de pepino pequeño $(n=3)$.

Nota. Chacón-Padilla y Monge-Pérez, 2020, a partir de los datos obtenidos.

\section{Conclusiones}

En los tres tipos de pepino, el número total de frutos por planta mostró una correlación positiva y altamente significativa, tanto con el rendimiento total como comercial, por lo que esta se debe considerar una característica esencial en el fitomejoramiento para el rendimiento en pepino.

Se concluye que el tipo de pepino, incluido en el análisis, influye de manera importante en el resultado de las correlaciones entre variables en esta hortaliza.

En pepino tipo largo, se halló una correlación negativa y altamente significativa entre el diámetro del fruto y el número total de frutos por planta y una correlación positiva y altamente significativa entre la longitud del fruto y el número total de frutos por planta. Estos resultados indican que, en este tipo de pepino, la selección de genotipos con frutos de menor diámetro y mayor longitud producirá un mayor número total de frutos por planta.

En pepino tipo pequeño, se obtuvo una correlación negativa y altamente significativa entre el diámetro del fruto y el porcentaje de sólidos solubles totales.

\section{Agradecimientos}

Los autores agradecen el financiamiento recibido por parte de CONARE, así como de la Universidad de Costa Rica, para la realización de este trabajo. Asimismo, agradecen la colaboración de Julio Vega, Andrés Oviedo y Carlos González en el trabajo de campo y de Mario Monge en la revisión de la traducción del resumen al idioma inglés.

\section{Referencias}

Afangideh, U., \& Uyoh, E. A. (2007). Genetic variability and correlation studies in some varieties of cucumber (Cucumis sativus L.). Jordan Journal of Agricultural Sciences, 3(4), 376-384.

Ahirwar, C. S., Singh, D. K., \& Kushwaha, M. L. (2017). Assessment of genetic variation in cucumber (Cucumis sativus L.) germplasm on correlation, path analysis and cluster analysis. Chemical Science Review and Letters, 6(23), 1886-1893.

Cancaya, S., Balkaya, A., \& Karaagac, O. (2010). Canonical correlation analysis for the determi- 
nation of relationships between plant characters and yield components in red pepper [Capsicum annuum L. var. conoides (Mill.) Irish] genotypes. Spanish Journal of Agricultural Research, 8(1), 67-73.

Deepa, S. K., Hadimani, H. P., Hanchinamani, C. N., Shet, R., Koulgi, S., \& Ashok. (2018). Studies on character association in cucumber (Cucumis sativus L.). International Journal of Current Microbiology and Applied Sciences, 7(11), 1977-1982.

Ene, C. O., Ogbonna, P. E., Agbo, C. U., \& Chukwudi, U. P. (2016). Evaluation of sixteen cucumber (Cucumis sativus L.) genotypes in derived savannah environment using path coefficient analysis. Notulae Scientia Biologicae, 8(1), 85-92.

Golabadi, M., Golkar, P., \& Eghtedary, A. (2015). Combining ability analysis of fruit yield and morphological traits in greenhouse cucumber (Cucumis sativus L.). Canadian Journal of Plant Science, 95, 377-385.

Hanchinamani, C. N. (2006). Genetic variability, divergence, heterosis and combining ability studies in cucumber (Cucumis satious L.). College of Agriculture, Department of Horticulture. Dharwad, India: University of Agricultural Sciences.

Kumari, A., Singh, A. K., Moharana, D. P., Kumar, A., \& Kumar, N. (2018). Character relationship and path coefficient analysis for yield and yield components in diverse genotypes of cucumber (Cucumis sativus L.). The Pharma Innovation Journal, 7(5), 33-38.

Madosa, E., Ciulca, S., Velicevici, G., Avadanei, C., Sasu, L., Cioroga, A., \& Friskan, I. (2008). Study of correlations between component characters of production capacity of sweet pepper (Capsicum annuum L. var. grossum). Bulletin UASVM, Horticulture, 65(1), 90-94.

Nwofia, G. E., Amajuoyi, A. N., \& Mbah, E. U. (2015). Response of three cucumber varieties
(Cucumis sativus L.) to planting season and NPK fertilizer rates in lowland humid tropics: sex expression, yield and inter-relationships between yield and associated traits. International Journal of Agriculture and Forestry, 5(1), 30-37.

Pal, S., Sharma, H. R., Das, A., \& Pandav, A. K. (2017). Character association and path analysis for fruit yield and it's contributing traits in cucumber (Cucumis sativus L.). International Journal of Agriculture, Environment and Biotechnology, 10(2), 163-170.

Sharma, S., Kumar, R., Chatterjee, S., \& Sharma, H. R. (2018). Correlation and path analysis studies for yield and its attributes in cucumber (Cucumis sativus L.). International Journal of Chemical Studies, 6(2), 2045-2048.

Shet, R. M., Shantappa, T., Ashok, \& Gurumurthy, S. B. (2018). Genetic variability and correlation studies for productivity traits in cucumber (Cucumis sativus L.). International Journal of Chemical Studies, 6(5), 236-238.

Soleimani, A., Ahmadikhah, A., \& Soleimani, S. (2009). Performance of different greenhouse cucumber cultivars (Cucumis sativus L.) in southern Iran. African Journal of Biotechnology, 8(17), 4077-4083.

Ullah, M. Z., Hasan, M. J., Chowdhury, A. Z., Saki, A. I., \& Rahman, A. H. (2012). Genetic variability and correlation in exotic cucumber (Cucumis sativus L.) varieties. Bangladesh Journal of Plant Breeding and Genetics, 25(1), 17-23.

Veena, R., Sidhu, A. S., Pitchaimuthu, M., \& Souravi, K. (2013). Character association for fruit yield and yield traits in Cucumber (Cucumis sativus L.). Electronic Journal of Plant Breeding, $4(1), 1108-1112$. 